\title{
Percepción comunitaria sobre la extensión forestal para la conservación de la cuenca del río Toa, Cuba
}

\author{
Yonny Martínez López ', Luis Andrei González Gomes 2, Emilio Martínez Rodríguez ${ }^{3}$, Juarez Benigno Paes ', \\ Fermín Correa Méndez ${ }^{4}$ \& Luciana Ferreira da Silva ${ }^{1}$
}

1. Departamento de Ciencias Forestales y de la Madera, Universidad Federal de Espírito Santo, Avenida Gobernador Lindemberg, 316, Centro, Jerônimo Monteiro, Espírito Santo, Brasil; yohnkar@gmail.com

2. Empresa Forestal Integral Baracoa, Departamento de Silvicultura, Reparto Bohorque, No. 126, Baracoa, Guantánamo, Cuba; ftalbcoa@enet.cu

3. Estación Experimental Agroforestal Baracoa, Departamento Forestal, Km 13 carretera a Guantánamo, Guantánamo, Cuba; eeafbaracoa@ forestales.co.cu

4. Departamento de Desarrollo Sostenible, Universidad Indígena de Michoacan, Carretera Pátzcuaro Erongaricuaro Kilómetro 3, Centro, Pátzcuaro, Michoacán, México; correa.mendez.fermin@gmail.com

Recibido 25-VII-2017 • Corregido 24-IX-2017 • Aceptado 30-X-2017

\begin{abstract}
Communal perception of forestry extension for the conservation of the Toa river basin, Cuba. The Toa River basin is one of the main reserves for biodiversity and natural resources in Cuba. We studied the communal opinion about the extension programs designed to mitigate overexploitation currently affecting the basin. Our interviews and surveys found that there farmers and producers know little about forest extension methods, that current land use favors erosion, and that pollution originates in nearby towns and industries.
\end{abstract}

Key words: conservation, basin, biodiversity, soil uses, forest practices
RESUMEN: La cuenca del río Toa es una de las principales reservas de biodiversidad y recursos naturales en Cuba. Estudiamos la opinión comunal sobre los programas de extensión diseñados para mitigar la sobreexplotación que actualmente afecta a la cuenca. Nuestras entrevistas y encuestas descubrieron que los agricultores y productores saben poco sobre los métodos de extensión forestal, que el uso actual de la tierra favorece la erosión y que la contaminación se origina en las ciudades e industrias cercanas.

Palabras clave: conservación, cuenca, biodiversidade, uso del suelo, prácticas forestales
La extensión forestal permite adquirir nuevos conocimientos para la conservación y protección sostenible de los recursos naturales y ecosistemas forestales, estas acciones contribuyen a mejorar las condiciones de vida y satisfacer las necesidades sociales (FAO, 1995). Según Donovan (2007) dichas acciones forman parte de la labor del extensionista, ya que le permite demostrar al productor, mediante el conocimiento o la práctica, la factibilidad de mejorar las actividades forestales. Los métodos de extensión forestal constituyen una herramienta fundamental para conjugar conocimientos y manejo de recursos naturales con la experiencia del campesino, transformando malas prácticas forestales en un mejor manejo.

Uno de los factores más importantes para mitigar las malas prácticas forestales es una buena comunicación, con intercambio de experiencias, conocimientos y pensamientos prácticos, venciendo contradicciones y la "resistencia al cambio" (Izco y Burneo, 2003).

En Cuba la extensión forestal se realiza mediante una fuerte interrelación entre las diferentes instituciones de investigación, centros de educación y otras instituciones de ciencia y tecnología para alcanzar el desarrollo forestal sostenible. Se considera que cualquier estrategia tiene que orientarse al intercambio entre los productores como un mecanismo indispensable para mejorar sus condiciones de vida y una mejor conservación de los recursos naturales (Cárdenas, Caballero, Martínez, Palenzuela \& Sáez, 2006).

La implementación de los métodos de extensión forestal permite alcanzar cambios en el accionar de los productores a partir de su capacitación, y de esa manera 
lograr que se adopten las tecnologías e innovaciones realizadas tanto por los centros de investigación como por otros productores (Geilfus, 2005). Estos nuevos conocimientos contribuyen a mejorar la acción de campesinos y productores, mediante las buenas prácticas forestales, que actualmente constituyen una amenaza para la protección de ecosistemas forestales como cuencas hidrográficas. En esta investigación se realizó una evaluación de los métodos de extensión forestal para mitigar la antropización de los recursos naturales de la cuenca del río Toa, Cuba.

\section{MÉTODOS}

Cuenca del río Toa: Tiene su origen en la vertiente norte, límite entre las provincias Holguín y Guantánamo, al este de la Sierra del Maquey. Constituye el límite municipal entre San Antonio del Sur, Yateras y Baracoa. La longitud es de $116,2 \mathrm{Km}$, su altura media fluctúa en los $488 \mathrm{msnm}$, una pendiente de $4,76 \%$, y un escurrimiento medio de $56,6 \mathrm{~m}^{3} \mathrm{~s}$.

La investigación se desarrolló siguiendo el cauce del río Toa, considerando su trayectoria desde la desembocadura hasta la comunidad de Quivijan (Fig. 1). Disponiendo de un trayecto de $14 \mathrm{Km}$ de río. Para el estudio se tuvo en cuenta ambos lados del río incluyendo el bosque de ribera, abarcando un área de $3360000 \mathrm{~m}^{2}$. Para la identificación de las especies fueron realizados cuatro transeptos de $30 \mathrm{~m}$ cada uno, los cuales consideraban desde la margen del río hacia arriba de la cuenca.

Métodos de extensión aplicados: Fueron empleados diversos métodos de extensión, como el día de campo-diagnóstico participativo, esta combinación permitió vincular experiencias del grupo técnico con el conocimiento de los campesinos. Se complementó el diagnóstico a partir de la técnica participativa mediante talleres, visitas, encuestas, y recorridos para determinar la veracidad de la información dada.

Encuestas: Se realizó una encuesta semi-estructurada, para identificar la información para alcanzar una proyección más cercana de la realidad del área objeto de estudio. Fueron incluidos los pobladores de las comunidades encontradas en todo el trayecto evaluado, miembros de cooperativas y campesinos de las comunidades. La población considerada estuvo representada por 180 personas ( 90 productores, sus familias) con un estimado de 30 personas por comunidad, localizados en toda la cuenca del río Toa quienes se dedican a la actividad agrícola, ganadera y forestal.

\section{RESULTADOS}

Los resultados de la encuesta indican que se percibe una mayor disponibilidad de leña y agua potable, seguido

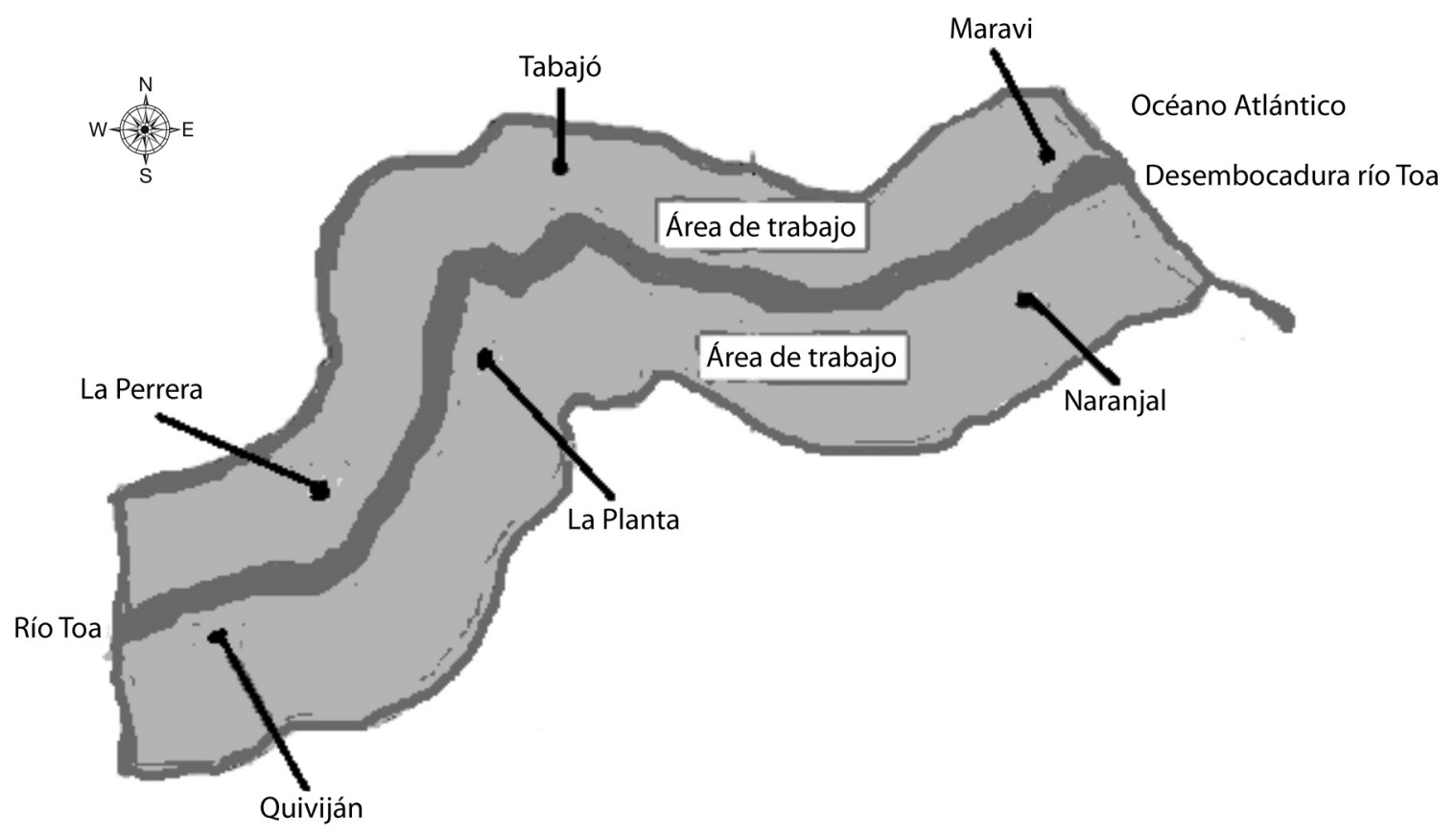

Fig. 1. Croquis demográfico y representativo del área de trabajo. 
de pasto, madera y suelo respectivamente (Cuadro 1), la información fue obtenida según los criteríos de personas que están vinculadas al trabajo productivo dentro de la cuenca, como representantes de las familias, los cuales utilizan estos recursos naturales para satisfacer sus principales necesidades y mejorar sus condiciones de vida.

\section{CUADRO 1}

Matriz de evaluación de los recursos naturales, en función de la disponibilidad y calidad en las comunidades estudiadas.

\begin{tabular}{|c|c|c|c|c|}
\hline \multirow[t]{2}{*}{ Recursos } & \multicolumn{2}{|c|}{$\begin{array}{l}\text { Hay suficiente } \\
\text { para todos (\%) }\end{array}$} & \multicolumn{2}{|c|}{ Calidad (\%) } \\
\hline & $\mathrm{Si}$ & No & Buena & Mala \\
\hline Agua potable & 51,2 & 48,8 & 51,6 & 48,4 \\
\hline Leña & 51,2 & 48,8 & 38,3 & 61,7 \\
\hline Madera & 36,1 & 63,9 & 30,0 & 70,0 \\
\hline Pasto & 42,2 & 57,8 & 41,6 & 58,4 \\
\hline Suelo & 31,6 & 68,4 & 16,1 & 83,9 \\
\hline
\end{tabular}

La densidad de uso del suelo permitió determinar que entre los cultivos varíos, frutales y forestales presentan una reacción inversa a como debe ser usado el suelo (Fig. 2), principalmente en la margen del río. El estudio comprendido posibilitó determinar además las principales especies que predominan a cada lado del río, según los transeptos estudiados (Cuadro 2).

Un resultado importante alcanzado a partir de los métodos de extensión forestal en el área de estudio fue el incremento de la capacitación referida a la temática de los suelos, profundizándose en los aspectos de conservación y mejoramiento, corrección de cárcavas, agricultura urbana, manejo de ganado, agricultura orgánica, floricultura, planificación y uso de la tierra.

Las medidas implementadas posibilitan el trabajo de la educación ambiental a todas aquellas personas que de una u otra forma tengan que ver con la misma, basado fundamentalmente en materiales educativos, mensajes educativos, programas de desarrollo cultural entre otras actividades sociales. Sensibilizando la interacción con los tenentes de tierras y dotarlos de conocimientos en materia de conservación y protección del medio ambiente con énfasis en los problemas ambientales tales como la contaminación de los cursos de agua.

\section{DISCUSIÓN}

El análisis realizado permitió determinar que para los recursos naturales agua potable y leña más del $50 \%$ de los encuestados manifiestan satisfacción. Esto puede estar dado por la cercanía del afluente de agua, además de ser este río el más caudaloso del país. No en todas las comunidades la calidad del agua es buena debido a que fueron detectados focos de contaminación con vertimiento directo al río, siendo las más afectadas las comunidades de La Perrera y La Planta. Sin embargo, para las comunidades de Maravi, Naranjal y Tabajó que están ubicadas más próximas a la desembocadura del río la leña que utilizan es obtenida de pequeños arbustos y árboles caídos principalmente en las márgenes del río.

La demanda de este recurso natural es mucho mayor debido a que en estas comunidades se concentran los mayores acentos de comunitaríos. De igual forma para

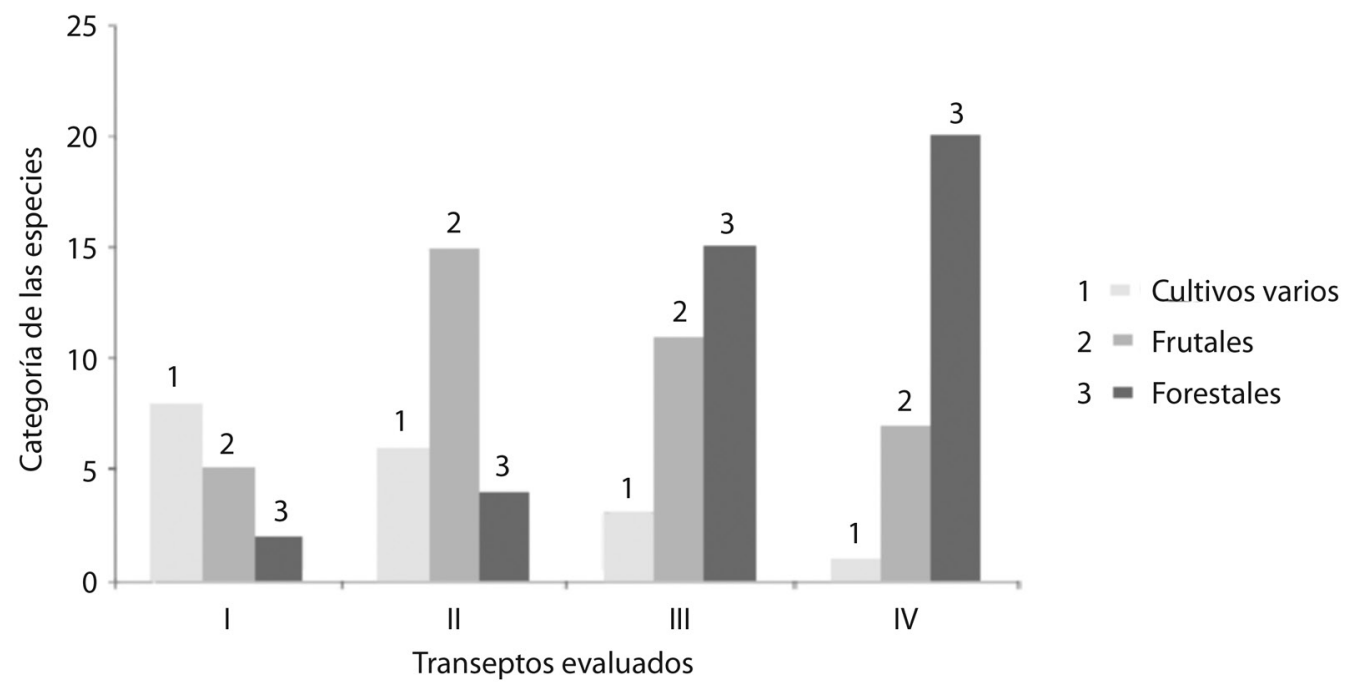

Fig. 2. Representación de las categorías de especies identificadas. 
CUADRO 2

Caracterización de la vegetación identificada en el área de estudio.

\begin{tabular}{|c|c|c|c|c|}
\hline \multirow{2}{*}{ Especie } & \multirow{2}{*}{ Nombre común } & \multirow{2}{*}{ Familia } & \multicolumn{2}{|c|}{ Lado } \\
\hline & & & Derecho & Izquierdo \\
\hline Artocarpus incisa L. & Guapén & Moraceae & 8 & 15 \\
\hline Bambusa vulgaris Schrad. ex J.C.Wendl. & Bambú & Poaceae & 13 & 10 \\
\hline Búcida buceras Grignon & Júcaro & Combretaceae & 8 & 10 \\
\hline Calophyllum antillanum Britton. & Ocuje & Cluseaceae & 35 & 33 \\
\hline Cecropia peltata L. Sist. & Yagruma & Moraceae & 6 & 4 \\
\hline Cedrela odorata L. Syn. & Cedro & Maliaceae & 10 & 6 \\
\hline Citrus sinensis L. Osbeck. & Naranjo & Rutaceae & 32 & 28 \\
\hline Clusia rosea Jacq. & Copey & Cluseaceae & 29 & 16 \\
\hline Coffea arabica L. & Cafeto & Rubiaceae & 54 & 43 \\
\hline Cocos nucifera L. & Cocotero & Arecaceae & 65 & 73 \\
\hline Cupania glabra SW. & Guara & Sapindaceae & 3 & 4 \\
\hline Chrisophyllum oliviforme L. & Caimitillo & Sapotaceae & 5 & 9 \\
\hline Ficus trigonata Pittier. & Jaguey & Moraceae & 4 & 6 \\
\hline Copaifera hymenaefolia Moric.* & Caguairán & Caesalpinaceae & 2 & 1 \\
\hline Gliricidia sepium Jacquin. & Júpiter & Fabaceae & 10 & 5 \\
\hline Grevillea robusta A. Cunn. & Roble australiano & Proteaceae & 4 & 5 \\
\hline Guarea guara Jacq. & Yamagua & Meliaceae & 5 & 30 \\
\hline Guazuma tomentosa Kunth. & Guásima & Sterculiaceae & 11 & 8 \\
\hline Jambosa vulgaris DC. & Pomarrosa & Myrtaceae & 12 & 2 \\
\hline Juniperus lucayana Britton.* & Sabina de olor & Cupresaceae & 3 & 1 \\
\hline Lonchocarpus domingensis Turpin. & Guamá de soga & Faraceae & 22 & 3 \\
\hline Manguifera índica L. & Mango & Anacardiaceae & 16 & 20 \\
\hline Manilkara albescens Griseb.* & Ácana & Sapotaceae & 2 & 4 \\
\hline Melicoccus bijugatus Jacq. & Mamoncillo & Sapindaceae & 24 & 32 \\
\hline Protium cubense Rose.* & Copal & Burseraceae & 3 & 6 \\
\hline Pera ekmanii Urb.* & Jiquí & Euphorbiaceae & 3 & 2 \\
\hline Pouteria mammosa L. & Sapote & Sapotaceae & 17 & 10 \\
\hline Prunus occidentalis SW.* & Cuajaní & Rosaceae & 4 & 2 \\
\hline Roystonea regia Kunth. & Palma real & Arecaceae & 14 & 36 \\
\hline Samanea saman Jacq. & Algarrobo del país & Mimosaceae & 15 & 20 \\
\hline Spondias mombin L. & Jaboncillo & Sapindaceae & 7 & 4 \\
\hline Talauma minor Urb.* & Azulejo & Magnoliaceae & 3 & 5 \\
\hline Tabebuia angustata Britton. & Roble blanco & Bignonaceae & 8 & 9 \\
\hline Taliparitis elatum SW. & Majagua & Malvaceae & 7 & 16 \\
\hline Terminalia catappa L. & Almendra & Combretaceae & 20 & 19 \\
\hline Theobroma cacao L. & Cacao & Malvaceae & 158 & 89 \\
\hline Zanthoxylon martinicense L. & Ayúa & Rutaceae & 14 & 4 \\
\hline Total & & & 260 & 249 \\
\hline
\end{tabular}

* Especies amenazadas y protegidas. 
el uso de madera para la construcción de casas y ranchos para la cría de animales. La carencia de especies forestales hace que el recurso leña y madera se ven afectados. Según estudios realizados por Garibaldi (2008) este análisis contribuye a encaminar un plan de acciones que posibilite mitigar la sobreexplotación de estos recursos, el cual posibilita encontrar respuestas para los comunitaríos de forma sostenible.

Estudios realizados por Begué, Perez, Joubert, Guarat y Zabala. (2014) plantean que en esta región del país predominan suelos ferralítico rojo típico (IIA), y suelos aluviales, de textura loan arcilloso y de estructura granular, pertenece a la clase agrológica III, no segmentados, ligeramente plásticos, estas características posibilitan la cosecha de plantas para la producción de alimentos de los campesinos y productores estatales.

Predominan pendientes alomadas y erosión fuerte, la que se agrava por las abundantes precipitaciones de la zona. El drenaje de forma general se evalúa de regular por su topografía, siendo el drenaje superficial excesivo y el interno bueno, es por ello que el daño detectado en las márgenes del río está determinado por la preferencia de cultivo de especies poco consistentes como el plátano y otros cultivos varíos para la producción de alimentos.

El uso de los suelos está representado por una flora muy variada, con una elevada concentración de especies, su endemismo está condicionado por la antigüedad del territorío, el cual está considerado como el mayor centro de diversificación de la flora de latifolia en Cuba, y contribuye con la riqueza florística de la región.

Algunas especies están amenazadas por su interés en la madera, principalmente las que tienen valor económico, como el ácana ( $M$. albescens), incienso (Protium fragans), azulejo (T. minor), cuajaní (P. occidentalis), jiquí (Pera ovalifolia), caguairán (G. hymenifolia), sabina de olor (J. lucayana), entre otras (Reyes \& Acosta, 2005). Estas especies son muy cotizadas y dada sus propiedades los campesinos la utilizan para construir sus viviendas, entre otros valores como medicinal y otros usos que los pobladores le confieren (Renda, Herrero \& Calzadilla, 2000).

Las familias con mayor número de especies, subespecies y/o variedades son: Rubiaceae, Asteraceae, Orchidaceae, Euphorbiaceae, Melastomataceae, Fabaceae y Myrtaceae. Teniendo en cuenta las características edafoclimáticas de la cuenca la convierten en un área proclive para la proliferación de las especies exóticas e invasoras de la flora (Mitjans, 2012; Oviedo, Herrera, Caluff, Regalado \& Ventosa, 2012), estas condiciones favorecen el incremento de la biodiversidad de especies, por lo que fueron identificadas 509 especies entre exóticas e invasoras.
Se identificó las preferencias de los comunitaríos en cuanto al aprovechamiento de los suelos cultivables (Fig. 2). Las especies clasificadas como cultivos varíos (producción de alimentos); frutales; y forestales, fueron localizadas en ambos lados del río principalmente el primer transepto evaluado. Según Renda (2005) y Rodríguez y Sánchez (2005), similares resultados fueron reportados en otras regiones de la cuenca del río Toa, con una tendencia diferente sobre la distribución de la flora en las márgenes del río, esta situación se agrava en el área de estudio debido a la presencia de varias comunidades, lo cual la antropización influye directamente sobre esos recursos de forma insostenible.

La tendencia al cultivo de alimentos y frutales desde los primeros metros de la faja hidrorreguladora (orilla del río) hacia arriba de la cuenca atente contra la protección del suelo. Es recomendable establecer plantaciones de especies tolerantes a la humedad con un marco de plantación apropiado ( $2 \mathrm{~m} \times 1,5 \mathrm{~m}$ a tres bolillos) que permita enriquecer esta faja del río, mediante intercalamiento con especies como Geoffroea inermis, Lonchocarpus dominguensis, Calophyllum antillanun Britt, Taliparitis etatum. Esta acción contribuye de forma sostenible con el uso de la tierra y el sistema de corriente de agua, cuya extensión está determinada por la calidad del agua de la cuenca (Hamilton et al., 2009).

Se evidencia que los suelos existentes a orillas del río $(30 \mathrm{~m})$ están proclives a su degradación, además del arrastre que provocan las crecientes de río Toa. Las actividades de reforestación contribuyen a mitigar este fenómeno, permitiendo retener el suelo, de igual forma que mejora su fertilidad y evita la erosión. Según Jiménez, Calzadilla y Renda, (2004) se estima que esta actividad debe representar más del $50 \%$ del área de la cuenca, aspecto que no se cumple en el área de estudio, donde en esta parte tan vulnerable del río solo se identifican árboles aislados o dispersos en las franjas hidrorreguladoras.

Según Renda (2013) la relación de las actividades agrícolas con los impactos que estas labores provocan en las cuencas hidrográficas es un fenómeno que afecta más del $40 \%$ de los suelos cubanos, manifestándose con mayor fuerza en las cuencas que se dedican a estos tipos de cultivos, donde la susceptibilidad de los tipos de suelos y su mal manejo cobra especial énfasis, aun cuando sus consecuencias o impactos negativos, en el escenarío agrarío, son previsibles en los tiempos actuales.

Los métodos de extensión forestal posibilitan identificar el estado de conservación de los recursos naturales existentes en el área de estudio, así como alertar a los comunitaríos, empresas y entidades competentes a tomar 
decisiones dirigidas a mejorar las prácticas forestales y contribuir a la protección y conservación de la cuenca.

Existe una tendencia inversa de uso del suelo que influye en la erosión de los mismos ocasionadas por inundaciones frecuentes en esta región del país debido a las precipitaciones acumuladas cada año.

Existen pocos conocimientos por parte de los comunitaríos y entidades productivas que limitan el desarrollo del programa de extensión forestal en el área de estudio.

Se detectó que a pesar de existir agua suficiente es importante controlar los focos de contaminaciones existentes en algunas comunidades como La Perrera y La Planta, así como otros vertimientos de productos forestales provenientes de la industria forestal.

\section{AGRADECIMIENTOS}

Los autores agradecen la colaboración de la Empresa Forestal Integral Baracoa y a los funcionaríos del Parque Nacional Alejandro de Humbolt, Cuba.

\section{REFERENCIAS}

Begué, G., Perez, H., Joubert, Y., Guarat, R., \& Zabala, B. (2014). Plan de manejo área protegida de recursos manejados Cuchillas delToa (2014-2020). Guantánamo, Cuba:Unidad de Servicios Ambientales Alejandro de Humboldt.

Cárdenas, I., Caballero, R., Martínez, M. M., Palenzuela, L., \& Sáez, A. (2006). El extensionista, promotor del desarrollo forestal sostenible. La Habana, Cuba: Dirección Forestal MINAGRI.

Donovan, D. G. (2007). El agua, los bosques y el informe mundial sobre el desarrollo de los recursos hídricos. Unasylva, 229(58), 62-63.

FAO (Food and Agriculture Organization of the United Nations). (1995). Evaluación de los recursos forestales 1990. Síntesis Mundial. Roma, Italia. (Estudio FAO: Montes N 124).

Garibaldi, C. (2008). Efectos de la extracción y uso tradicional de la tierra sobre la estructura y dinámica de bosques fragmentados en la Península de Azuero, Panamá. Tesis de Doctorado en Ciencias Forestales. Centro de Estudio
Forestal, Facultad Agroforestal, Universidad de Pinar del Río, Pinar del Río, Cuba.

Geilfus, F. (2005). 80 herramientas para el desarrollo participativo: diagnóstico, planificación monitoreo, evaluación. San Salvador, El Salvador: IICA.

Hamilton, L. S., Dudley, N., Greminger, G., Hassan, N., Lamb, D., Stolton, S., \& Tognetti, S. (2009). Estudio temático elaborado en el ámbito de la evaluación de los recursos forestales mundiales 2005. Los bosques y el agua. Roma, Italia. (Estudio FAO: Montes $\mathrm{N}^{\circ} 155$ ).

Izco, X., \& Burneo, D. (2008). Herramientas para la valoración y el manejo forestal sostenible de los bosques sudamericanos. Programa de Conservación de Bosques. Quito, Ecuador: UICN.

Jiménez, M., Calzadilla, A., \& Renda, A. (2004). La agroforestería: una opción para el desarrollo sostenible. Revista forestal Baracoa, 1, 57-63.

Mitjans, B. (2012). Rehabilitación del bosque de ribera del río cuyaguateje, en su curso medio. Estrategia participativa para su implementación. Tesis de Doctorado. Centro de Estudio Forestal, Facultad Agroforestal, Universidad de Pinar del Río, Pinar del Río, Cuba.

Oviedo, R., Herrera, M., Caluff, L., Regalado, I., \& Ventosa, J. (2012). Lista nacional de especies de plantas invasoras y potencialmente invasoras en la república de Cuba. Bissea, 6(1), 22-96.

Renda, A., Herrero, J.A., \& Calzadilla, E. (2000). Papel de la vegetación forestal y los sistemas agroforestales en el manejo de cuencas hidrográficas. La Habana, Cuba: IIF. MINAG.

Renda, A. (2005). Manejo de suelos, bosques y agua en el escenarío agrarío. In II Encuentro Internacional de Jóvenes Investigadores Forestales DEFORS. La Habana, Cuba.

Renda, A. (2013). La vegetación forestal, los sistemas agroforestales y el manejo de cuencas hidrográficas en Cuba. La Habana, Cuba: INAF.

Reyes, O., \& Acosta, F. (2005). Vegetación en Cuba: Parque Nacional "Alejandro de Humboldt". Baracoa, Cuba. Rapid Biological Inventories. p54-69 (Report N¹4).

Rodríguez, R., \& Sánchez, J. (2005). Diseño sostenible para la recuperación y conservación de las fajas forestales hidrorreguladoras del Río Toa. Guantánamo. In II Encuentro Internacional de Jóvenes Investigadores Forestales DEFORS. La Habana, Cuba. 